\title{
Comparison of management options for patients with acoustic neuromas
}

\author{
Douglas Kondziolka, M.D., M.Sc., F.R.C.S.(C), L. Dade LunSford, M.D., \\ AND JOHn C. FliCKInGER, M.D. \\ Departments of Neurological Surgery and Radiation Oncology, and the Center for Image-Guided \\ Neurosurgery, University of Pittsburgh, Pittsburgh, Pennsylvania
}

\begin{abstract}
Management options for patients with vestibular schwannomas (acoustic neuromas) include observation, tumor resection, stereotactic radiosurgery, and fractionated radiotherapy. In this report the authors review their 15-year experience with radiosurgery and discuss indications and expectations in relation to the different approaches. They conducted a survey of neurosurgeons to determine management preferences in two different cases of intra- and extracanalicular tumor presentations. Patient decisions must be based on quality information derived from peer-reviewed literature.
\end{abstract}

\section{KEY WORDS • acoustic neuroma • vestibular schwannoma • radiosurgery • gamma knife}

For the treatment of patients with acoustic neuromas there are several options including observation, resection, SRS, and fractionated SRT. Many patients choose between SRS and resection based on their own specific goals and their understanding of possible results. The decision can be difficult and depends on the sources and strengths of information given to them. These include discussions with surgeons and other physicians, written material disseminated by peer-reviewed medical journals, brochures from support groups, internet-based reports (of variable reliability), and discussions among fellow patients. We believe that information provided in the peer-reviewed medical literature is the most reliable for patient education. Nevertheless, some patients become confused by what they perceive as conflicting opinions among physicians. Many such physicians provide only one kind of treatment and may appear to be biased toward their own approach.

Resection is indicated for patients harboring larger tumors that have caused major neurological deficits due to brain compression. Surgeons perform SRS for small- or medium-sized tumors, their goals being to preserve neu-

\footnotetext{
Abbreviations used in this paper: $\mathrm{CSF}=$ cerebrospinal fluid; $\mathrm{CT}=$ computerized tomography; GKS = gamma knife surgery; $\mathrm{MR}=$ magnetic resonance; NF2 = neurofibromatosis Type 2; $\mathrm{SRS}=$ stereotactic radiosurgery; SRT $=$ stereotactic radiotherapy; $\mathrm{VS}=$ vestibular schwannoma.
}

rological function and prevent tumor growth. The longterm outcomes of SRS, particularly GKS, have proven its role in the primary or adjuvant management of this tumor. Fractionated radiotherapy has been suggested as an alternative in selected patients with larger tumors for whom microsurgery may not be feasible, as well as in some patients in whom preservation of cranial nerve function is being attempted. Most such centers do not offer conformal SRS. Cases of NF2 pose specific challenges, particularly the preservation of hearing and other cranial nerve function.

Primary clinical issues include avoiding tumor-related or treatment-related mortality, prevention of further tumor-induced neurological disability, minimizing treatment risks such as CSF leakage, infections, or cardiopulmonary complications, maintaining regional cranial nerve function (facial, trigeminal, cochlear, and glossopharyngeal/vagal), avoiding hydrocephalus, maintaining quality of life and employment, and reducing cost. In choosing any particular therapy, the team should strive to meet all of these goals.

\section{MANAGEMENT OF ACOUSTIC NEUROMA}

\section{Resection of the Lesion}

There are three main surgical routes for resection of an acoustic neuroma. These approaches are chosen based on educational bias, surgery-related experience, and the spe- 
cific goals of the operation. The suboccipital retrosigmoid approach is the oldest and remains widely used, particularly when hearing preservation is attempted. The translabyrinthine approach destroys hearing but provides direct exposure to the tumor without requiring cerebellar retraction. Even large tumors can be removed through this route. The "middle fossa" approach is performed via a temporal craniotomy but requires elevation of the temporal lobe and drilling of the temporal bone to expose the auditory canal from above. Using this route, hearing preservation can be attempted. It is usually chosen for patients with intracanalicular tumors.

The goals and acceptable clinical outcomes have evolved over time. Many years ago, the goal was simple debulking of the tumor (which was often large) and relief of regional brainstem compression and hydrocephalus. The goal was life saving. Neurological deficits such as hearing loss, facial weakness, or balance disorders were tolerated as simply part of the expected result. In the 1970s, the introduction of the operating microscope facilitated meticulous dissection of the tumor, making possible attempts at cranial nerve preservation. Over the ensuing 20 years, preservation of facial nerve continuity became more common than not. During the 1990s, hearing preservation became an achievable goal in selected cases. At the same time, improvements in anesthesia-related technique and wound closure reduced the risk of cerebellar infarction, meningitis, and CSF leakage. Nevertheless, these problems still continue to exist and CSF remains a significant problem after resection. ${ }^{13}$

\section{Stereotactic Radiosurgery}

Stereotactic radiosurgery has become a common therapeutic choice for patients with acoustic tumors (VSs). ${ }^{11,20}$ Experience with radiosurgery now extends more than 30 years. During the late 1980s and early 1990s, patients and their doctors chose radiosurgery or resection based mainly on early outcomes data derived from limited patient series. ${ }^{6-10,23-25}$ In 1987 we began a prospective assessment of the response of patients with acoustic tumors to GKS. Both early and later (5-10-year) outcomes were determined by obtaining and evaluating serial neuroimaging, hearing and facial function, and physician-based studies. ${ }^{17}$ Because expected outcomes may vary for patients with solitary tumors and those with NF2, we have analyzed data in these patient populations separately.

Patient Characteristics: University of Pittsburgh. Eight hundred twenty-seven patients underwent SRS for an acoustic tumor (VS) at the University of Pittsburgh during a 15-year interval. These included 765 patients with solitary tumors and 62 with NF2. Fifty percent of the tumors were left sided and 50\% were right sided. Fifty percent of patients were female. The patients ranged in age from 12 to 95 years (mean 56 years).

A resection had been performed in 165 patients (20\%). Twenty-nine patients had undergone two prior resections, eight underwent three resections, and four underwent four resections. Most patients exhibited normal facial function (House-Brackmann Grade I). ${ }^{15}$ The Gardner-Robertson Scale was used to code hearing function. ${ }^{12}$ "Useful" hearing before SRS was noted by $33 \%$ of patients.

In our last review of 45 patients with NF2, prior resec- tion had been performed in $13,{ }^{35}$ multiple resections had been performed in four patients. Normal facial function before SRS was present in 74\%, normal trigeminal function in $75 \%$, and useful hearing (Gardner-Robertson Grades I and II) in $31 \%$.

Technique of GKS. All patients underwent stereotactic GKS (Elekta Instruments, Atlanta, GA) after administration of a local anesthetic and intravenous sedation when necessary. Children younger than the age of 12 years with NF2 underwent SRS after induction of general anesthesia. Radiosurgery was performed with the assistance of CT scanning between 1987 and 1991. Subsequently MR imaging was the modality of choice after a prospective comparison study confirmed the accuracy of MR imagingbased stereotactic targeting. ${ }^{16}$ Multiple isocenters were used to conform the radiation margin to the intra- and extracanalicular tumor components (Fig. 1). ${ }^{10}$ The $50 \%$ isodose line was used to cover the tumor margin in $88 \%$ of patients with solitary tumors. An initial tumor margin dose of 18 to 20 Gy was selected based on the initial experience reported by the Karolinska group. ${ }^{23}$ This dose was decreased to 16 to 18 Gy within the first 2 years and by 1992 was decreased further to a margin dose of 14 to 16 Gy. Repeated reevaluations of the cranial nerve response prompted further small decreases in dose to preserve cranial nerve function. ${ }^{7,10}$ The mean dose delivered to the margin in cases of both non-NF2 and NF2 tumors was 14 Gy, although the most commonly prescribed margin dose at the present time is $13 \mathrm{~Gy}$, and this dose has been fairly constant for the past 9 years. ${ }^{19,35}$ Dose selection in individual patients was based on the following factors: tumor volume, history of surgery, hearing status, facial motor function, and patient wishes. After SRS, all patients received a single 40-mg dose of intravenous methylprednisolone and were discharged from hospital the next morning.

Follow-Up Evaluations. Serial imaging studies (MR imaging or CT scanning when MR imaging was contraindicated) were requested every 6 months for the first 2 years, annually for the next 2 years, and then biannually. Serial audiograms were obtained at 6 to 12 -month intervals in patients with preserved hearing. Contrast-enhanced imaging studies were conducted to define the tumor response and to identify any peritumoral changes. Before and after SRS, each tumor was measured in five separate dimensions (three extracanalicular and two intracanalicular) by using a method previously reported. ${ }^{18} \mathrm{~A}$ significant imaging-documented change determined using this caliper technique was defined as a difference of $\pm 2 \mathrm{~mm}$.

The Long-Term Experience. We continue to evaluate a cohort of 162 patients who have undergone follow-up evaluations for at least 10 years since their procedure. This study represented results of our initial techniques. ${ }^{17}$ The majority of irradiated acoustic tumors $(\sim 70 \%)$ decreased in size over time (Fig. 1). In nine patients tumors increased in size and all were identified within the first 3 years after SRS. ${ }^{17}$ Enlargement of the lesion represented either true neoplastic tumor growth (four cases) or tumor death with an expansion of the tumor margins as the central portion of the tumor became necrotic. In the latter five patients subsequent imaging studies confirmed tumor volume regression. Four patients underwent resection. No 


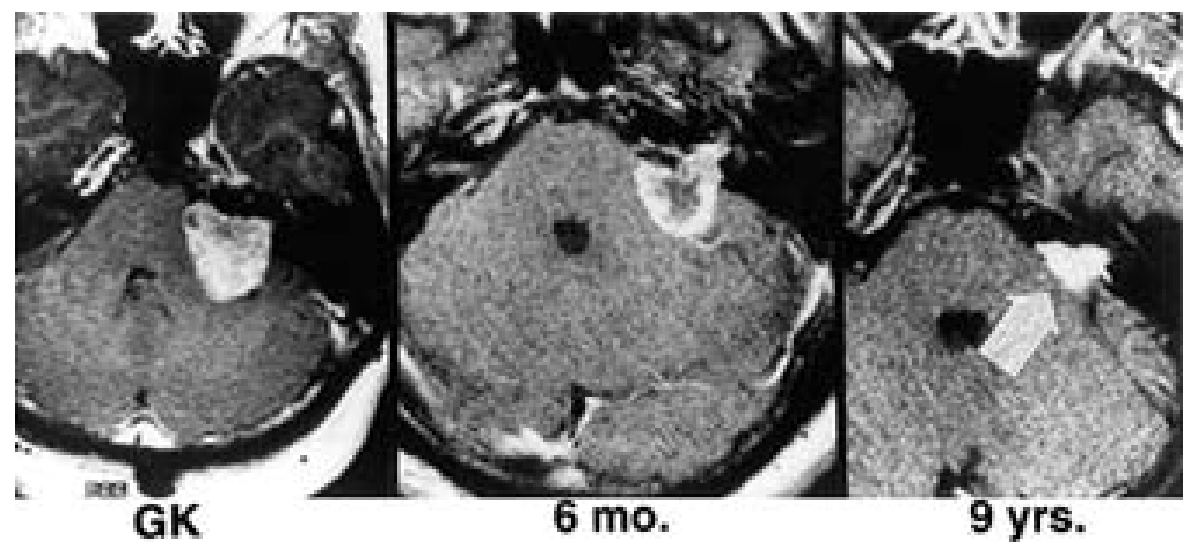

Fig. 1. Axial serial MR images obtained at SRS (left), 6 months posttreatment (center), and after 9 years of follow up (right). This 44-year-old man was treated with an 11-isocenter gamma knife plan to a margin dose of 14 Gy. He maintains normal facial nerve function and experienced a marked return of his energy within several months of the procedure.

further increase in tumor volume was identified in any patient at additional follow-up examination. ${ }^{7}$ Patients returned to their routine activities immediately. In our 5 to 10-year review, three patients developed hydrocephalus and required placement of a ventriculoperitoneal shunt. ${ }^{17}$ All new or worsened post-SRS deficits occurred within 28 months of the procedure, and no patient described a treatment-related problem after the 3rd year.

Current Experience With Solitary Tumors. Refinements in technique followed a continued review of results. In 1991 we began to use MR imaging-based stereotactic planning because CT scanning-based planning did not demonstrate well the intracanalicular portion of the tumor. With the former, we could visualize the tumor and regional neural structures in greater detail. This facilitated the use of multiple small irradiation isocenters for more conformal SRS. With this type of SRS, the rate of cranial nerve morbidity dropped precipitously. Similarly, our analysis of hearing preservation in patients with NF2 showed significant gains.

One hundred ninety-two patients underwent GKS between 1992 and 1997 and were eligible for extended follow-up reviews. ${ }^{7}$ The maximum follow-up interval in this cohort was 65 months. The median tumor margin dose was $13 \mathrm{~Gy}$. The actuarial 5-year clinical tumor control rate (defined as no need for additional treatment) was $97 \%$. One patient underwent a resection 6 months after radiosurgery. Five-year actuarial rates of developing any facial weakness, facial numbness, hearing level preservation, and preservation of testable speech discrimination were $1.1 \pm 0.8 \%, 2.6 \pm 1.2 \%$, and $71 \pm 4.7 \%$, and $91 \pm 2.6 \%$, respectively. At a tumor margin dose of $13 \mathrm{~Gy}$ or less, the rate of facial neuropathy was $0 \%$, whereas above 13 Gy it was $2.5 \%$ (usually mild and transient). The tumor diameter did not significantly affect results. The authors of studies from other centers have reported similar results. ${ }^{26}$

Neurofibromatosis Type 2. Serial neuroimaging studies of 45 tumors during a median 36-month follow up (range 6-120 months) demonstrated that 16 tumors $(36 \%)$ had regressed, 28 tumors $(62 \%)$ remained unchanged in size, and one tumor $(2 \%)$ had progressed. ${ }^{35}$ Loss of central contrast within the tumor was observed in some patients and thought to reflect tumor necrosis.
At our last detailed review, the mean clinical follow-up period was 41 months (range 6-120 months). No patients exhibited improvement on their clinical examination after GKS. In 30 patients $(67 \%)$ clinical status was maintained and in 15 patients $(33 \%)$ some degree of clinical deterioration was demonstrated. Two patients $(4 \%)$ died during the follow-up period of unrelated illnesses. The median Karnofsky Performance Scale score after GKS was 80. Thirty-five patients $(78 \%)$ were able to perform normal daily activities at the time of the last examination (Karnofsky Performance Scale score $\geq 80$ ).

Of the 14 tumors associated with useful hearing (Gardner-Robertson Grade I or II) at the time of SRS, six (43\%) demonstrated no change in hearing class during the follow-up period. Eight other patients lost all functional hearing (defined as absent speech discrimination) at a mean of 6 months after SRS (range 3-15 months). The overall rate of hearing preservation was $43 \%$. In 1992, we began to use MR imaging-guided stereotactic planning and increased numbers of smaller isocenters. By specifically dividing the population into patients treated prior to 1992 and those treated after 1992, the difference in hearing preservation again becomes apparent. Prior to 1992, five patients with useful hearing (Grade I or II) were treated. All patients subsequently lost speech discrimination. After 1992, nine patients with useful hearing underwent GKS. Six $(67 \%)$ of the patients had hearing preservation at the time of last examination. ${ }^{35}$ Thirty-one tumors (69\%) were associated with intact facial nerve function (HouseBrackmann Grade I) at the time of GKS. The overall rate of facial nerve preservation (Grade I) was $81 \%$. Thirty-six of the treated tumors were associated with intact trigeminal nerve function. Three patients (8\%) experienced trigeminal distribution sensory loss at a mean of 5 months (range 4-5 months) after radiosurgery. One patient subsequently recovered all trigeminal function, whereas residual deficits developed in two. The overall rate of trigeminal nerve preservation was $94 \%$.

Tumor Resection or SRS? There have been a number of patient-based surveys conducted to study outcomes after treatment for acoustic neuroma. One of the first was a survey of 541 patient members of the Acoustic Neuroma Association who provided data on tumor resection be- 
D. Kondziolka, L. D. Lunsford, and J. C. Flickinger

tween 1973 and $1983 .{ }^{36}$ Of these patients facial weakness was present in $62 \%$, eye-related problems in $84 \%$, depression in $38 \%$, sleep disturbance in $26 \%$, and speech or swallowing difficulties in $16 \%$. More recently a larger survey of 1579 cases in which resections were performed between 1989 and 1994 found improved results that included a $44 \%$ rate of facial weakness, an $11 \%$ rate of CSF leakage, and persistent balance problems after 1 year in $9 \%$. In approximately $8 \%$, recurrent or residual tumor was revealed on follow-up imaging. ${ }^{37}$

Better results following resection have been documented since that time. Samii and Matthies ${ }^{32}$ and Gormley, et al., ${ }^{13}$ found that complete tumor removal was a frequent outcome. Neurological and systemic morbidity, however, remained present with $1 \%$ mortality rates and CSF fistula rates of 9.2 and $15 \%$, respectively. Experienced surgical teams have reported significant reductions in postresection complication rates, although the incidence of specific problems such as CSF leakage have remained unchanged. ${ }^{3}$ Brennan, et al., ${ }^{3}$ reported that translabyrinthine approach-related leaks had a higher incidence of surgical repair than retrosigmoid approach-related leaks. For patients with large acoustic tumors $(>3 \mathrm{~cm}$ in extracanalicular diameter) and those with progressive neurological deficits that require brainstem decompression, surgical resection (total or subtotal) is the preferred option. We believe that a complete resection should be performed in such patients if possible, but not at the expense of sacrificing neurological function. Stereotactic radiosurgery can be considered for patients with intracanalicular, small- or medium-sized acoustic tumors because most such cases do not involve a rapidly progressive neurological syndrome. The initial symptoms caused by most acoustic tumors are not improved by resection. ${ }^{28}$

In a recent report, Martin, et al., ${ }^{21}$ evaluated quality of life in patients after tumor resection. They found a disparity between the patients' reports and the physicians' assessments of function, with decreases in physical functioning, general health, and social functioning after surgery. More severe balance functions led to worse social functioning (Table 1).

The long-term effects of both resection and SRS must be documented to assist in physician and patient decision making. Surprisingly little information has been published on long-term imaging-based outcomes after resection. Cerullo, et al., ${ }^{4}$ noted a $10 \%$ recurrence rate by 10 years following resection. Mazzoni, et al., ${ }^{22}$ reported a series of more than 100 patients in whom hearing preservation was attempted. The overall tumor recurrence rate was $8.1 \%$. Although these papers are often criticized for their results, they represent an honest evaluation of longer-term imaging results. All groups should strive to obtain serial imaging studies. Post, et al., ${ }^{30}$ found that in four $(7 \%)$ of 56 patients the resection was incomplete in their attempt at preserving hearing and that in three regrowth developed within 3 years. In the largest series, Samii and Matthies ${ }^{32}$ reported a complete resection in $98 \%$ of patients and documented recurrence in six of 880 without NF2. In our SRS series, $98 \%$ of the patients required no further surgery and in $94 \%$ there was imaging confirmation of persistent tumor control. Tumors that increased in size in the 1st or 2nd year after GKS did so usually in association with central tumor necrosis, with a small expansion of the tumor
TABLE 1

Comparison of patient-reported survey data on management of VSs

\begin{tabular}{lcc}
\hline \hline \multicolumn{1}{c}{ Issue } & Resection* & Radiosurgery $\dagger$ \\
\hline no. of cases & 32 & 11 \\
year of study & 2001 & 1998 \\
no change in employment & $68 \%$ & $69 \%$ \\
offers positive recommendation & $74 \%$ & $95 \%$ \\
mean tumor size (mm) & 25 & 22 \\
new balance problems & $78 \%$ & $7 \%$ \\
new-onset tinnitus & $57 \%$ & $3 \%$ \\
new facial nerve dysfunction & $63 \%$ & $10 \%$ \\
\hline
\end{tabular}

* As reported by Martin, et al.

$\dagger$ As reported by Kondziolka, et al., (patients managed from 1987-1992).

capsule. Most such tumors then regressed to a size smaller than that at baseline with longer follow up. Such transient expansion may be associated with transient retroauricular pain, perhaps due to regional dural inflammation. Recurrence or continued tumor growth may follow resection or SRS, ${ }^{27,29}$ and periodic neuroimaging studies should be obtained in all patients.

We believe that all patients with newly diagnosed, residual, or recurrent acoustic tumors $(<3 \mathrm{~cm}$ in extracanalicular diameter) are now suitable candidates for SRS. ${ }^{8,17}$ Larger tumors are not as good candidates because of the dose reduction necessary to reduce the rate of potential radiation-related side effects. Hearing preservation should be attempted in younger patients with good hearing, either with SRS or resection. ${ }^{31}$ In our first 3-year experience, we accepted elderly patients, those with concomitant medical problems that argued against resection, those with residual or recurrent tumors after resection, and those with preserved hearing function. By 1991 we began to offer radiosurgery to all patients with acoustic tumors regardless of age, surgical history, or symptoms. For older patients (age $>75$ years) with small and minimally symptomatic tumors, we continue to advocate observational serial imaging-based evaluation. ${ }^{2,33,39}$

When we evaluate patients with acoustic tumors, many ask the following two questions. First, is the tumor more difficult to resect if GKS fails? The answer to this is not clear. Few patients have required resection, and the opinions of the surgeons we have asked indicate that some tumors were less difficult, some about the same, and some more difficult. In a report on this issue that included 13 patients who had undergone resection after SRS, eight lesions were thought to be more difficult to treat. In five of these eight patients, however, resection failed before they underwent radiosurgery.

Second, patients inquire about the risk of delayed malignant transformation. Malignant schwannomas are rare, but have been reported de novo, after prior resection, ${ }^{14}$ and after irradiation. Our answer is that such a risk always exists after irradiation but that the risk should be very low. No such lesions have been documented in any of our 5400 patients during our first 15-year experience with SRS, but we tell the patients that the risk is between 1:1000 and $1: 20,000$. We reported on one patient with a malignant mesenchymal tumor of the cerebellopontine angle that resembled an acoustic tumor. ${ }^{5}$ The authors of a report from Japan found a malignant tumor 4 years after resec- 
tion and 6 months following SRS. The time interval after irradiation was too short to indicate causation. ${ }^{14}$ In a second report investigators noted the development of a temporal lobe glioblastoma 7.5 years after radiosurgery for a nearby acoustic neuroma. The temporal lobe had received a low radiation dose. ${ }^{34}$ In contrast, one of our patients underwent initial management of a frontal lobe astrocytoma and years later developed an acoustic neuroma. Was the development of these tumors related in some oncogenetic way or were they radiation related? We believe the risk of developing a tumor years after radiosurgery is much less than that of mortality immediately after resection, and likely less than the risk of developing another tumor in another body location.

Fractionated Radiotherapy. In the last several years, a number of groups have used fractionated radiotherapy to treat patients with acoustic neuromas. This technique was developed when surgeons at several centers that used LINAC-based irradiation technology were not satisfied with the results or accuracy of their device after singlefraction irradiation (SRS). To decrease the cranial nerve deficits, they began to deliver radiation over multiple sessions (fractionation). The goal of this approach is to weaken the effect of each radiation administration and to maintain brain or nerve function. Correspondingly, this also weakens the effect of the radiation on the tumor target. There are little data on this approach in the peer-reviewed literature that includes diligently-documented outcomes and follow-up data. Williams, et al., ${ }^{38}$ reported on 80 patients who underwent fractionated SRT. The median follow-up interval after radiotherapy was 2.9 years. Seventy patients received $25 \mathrm{~Gy}$ in five fractions and 10 received 30 Gy in 10 fractions. The treatment was delivered using CT targeting, which is limited in evaluating the intracanalicular portion of the tumor. In only 19 of 80 patients was Gardner-Robertson Grade I or II hearing present at the time of treatment. Hearing levels were preserved in $82 \%$ (actuarial data). Two patients experienced transient trigeminal neuropathies and no patient suffered a significant facial neuropathy. In a separate oral report, Lederman and associates (unpublished data) provided results from the Staten Island group at the 2001 meeting of the International Stereotactic Radiosurgery Society. They offered no treatment planning images, no cranial nerve outcome data derived using the accepted grading systems, and "hearing preservation" was not defined. They noted that hearing was preserved at a rate "above" $90 \%$, but the quality of hearing was not reported.

In a more comprehensive report, Andrews, et al., ${ }^{1}$ reported 69 patients who underwent GKS and 56 patients who underwent LINAC-based radiotherapy. Tumor control rates were high (97\%) in the early follow-up period, and the rate of cranial nerve deficits was low in both groups. Using their technique, they found a higher rate of early hearing preservation after radiotherapy, but both treatments were associated with a median follow-up time of less than 10 months. Their rate of hearing preservation after SRS $(33 \%)$ was lower than that reported by others. The main drawback of their report was the lack of randomization. Patients were allocated to either treatment according to "strong physician preferences."

Optimally, appropriate radiation doses should be delivered precisely to the tumor, and the regional brain struc- tures should be spared radiation. This is not the case when administering fractionated doses in which larger volumes of regional tissue are irradiated. We believe that any advantage in fractionation in limiting toxicity only makes sense if the target volume contains normal brain or nerve. Sophisticated stereotactic radiosurgical instruments allow regional brain or nerve to be spared because of framebased, single-session, image guidance. We do not believe that fractionation provides any useful advantage over radiosurgical techniques that have been used for the last 10 years.

Survey of Neurosurgeons on Acoustic Neuroma Management. A survey was mailed to members of the Congress of Neurological Surgeons in July 2002. Six hundred sixty-three surgeons (30\%) responded to the survey. There were four questions written on one page. Forty one percent of responders were between the age of 40 and 50 years (Table 2). Eighty percent of neurosurgeons (530) surveyed had either performed radiosurgery in a patient with an acoustic neuroma or had referred a patient for neurosurgery.

Survey Case One. Question: You are a 37-year-old neurosurgeon who presents with mild decreased hearing on one side. You have no tinnitus and no balance problems. Facial function is normal. An MR image reveals an intracanalicular acoustic neuroma and serial images have demonstrated a small amount of growth. Which management strategy would you choose for yourself? Observation; resection; SRS; or fractionated radiotherapy? (Fig. 2).

Response: The majority of surgeons (283 [43\%]) stated that they would choose SRS for management of their small acoustic tumor. Only 122 surgeons (18\%) stated that they would choose resection. Fractionated radiotherapy was chosen by $2 \%$ of responders. Interestingly, 240 surgeons $(36 \%)$ stated that they would continue to observe their tumor rather than undergo any specific treatment at the time. It had been stated in the case presentation that serial images had already demonstrated a small amount of growth. This tumor had been observed and was increasing in volume. Nevertheless, approximately one third of responders continued to choose observation for a 37-yearold patient with a small but growing tumor.

We evaluated the age of the responding surgeon and compared this to the treatment chosen by that surgeon (Table 2). Across the age groups between 30 and 70 years,

TABLE 2

Results of survey mailed to neurosurgeons

\begin{tabular}{lrrrrr}
\hline \hline & \multicolumn{5}{c}{ Surgeon Age Range in Years } \\
\cline { 2 - 6 } $\begin{array}{l}\text { Recommended } \\
\text { Treatment }\end{array}$ & $30-40$ & $40-50$ & $50-60$ & $60-70$ & $>70$ \\
\hline Case 1 & & & & & \\
$\quad$ observation & 45 & 106 & 64 & 24 & 1 \\
resection & 15 & 47 & 36 & 20 & 3 \\
$\quad$ radiosurgery & 60 & 104 & 74 & 42 & 3 \\
$\quad$ radiotherapy & 1 & 11 & 4 & 0 & 0 \\
Case 2 & & & & & \\
$\quad$ observation & 6 & 7 & 13 & 10 & 1 \\
resection & 69 & 148 & 92 & 36 & 2 \\
$\quad$ radiosurgery & 46 & 102 & 68 & 40 & 4 \\
$\quad$ radiotherapy & 1 & 12 & 5 & 0 & 0 \\
\hline
\end{tabular}




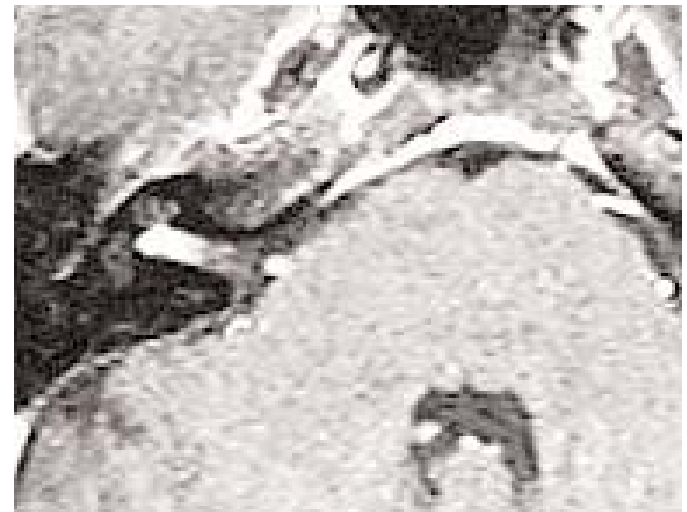

Fig. 2. Axial MR image obtained in a 37-year-old man with a right intracanalicular acoustic neuroma (Survey Case 1).

at least twice as many neurosurgeons chose SRS for their tumor rather than resection. This is most pronounced in the younger surgeon age group (30-40 years), in which the number of surgeons choosing SRS over resection was fourfold higher. Observation, however, continued to be chosen by many. Although one might think that an older person might choose radiosurgery over resection, simply to avoid the risks of general anesthesia or the surgical exposure, this did not necessarily appear to be true. This case reflected the care of an actual neurosurgeon who had undergone GKS. He remains well 18 months following his procedure, maintaining a full practice. He has experienced no facial weakness or change in hearing.

Survey Case Two. Question: You are a 50-year-old neurosurgeon who presents with mild decreased hearing on one side. You have tinnitus but no balance problems. Facial function is normal. An MR image reveals a left acoustic neuroma. Which management strategy would you choose for yourself? Observation; resection; SRS; or fractionated radiotherapy? (Fig. 3).

Response: In this scenario, the neurosurgeon had a medium-sized acoustic tumor that indented the middle cerebellar peduncle but did not compress the fourth ventricle. The tumor measured $22 \mathrm{~mm}$ in the maximum diameter. The minority of surgeons $(6 \%)$ recommended continued observation for a tumor of this size. Resection was recommended by 347 surgeons (52\%), whereas SRS was chosen by 261 surgeons (39\%). Fractionated radiotherapy was only chosen by $3 \%$. When the results were stratified by age, resection was the most popular choice across the groups between the age of 30 and 60 years. Radiosurgery, however, became more popular with advancing age of the survey group, passing resection as the most popular choice when the neurosurgeon was older than age 60 years. It appears that surgeons chose to undergo resection because of the larger volume of the tumor that indented the lateral surface of the brainstem. This patient was also a real neurosurgeon who had undergone SRS. He remains well 18 months after the procedure and the tumor has decreased in size. Facial function remains normal.

\section{CONCLUSIONS}

Patients with acoustic neuromas have several treatment

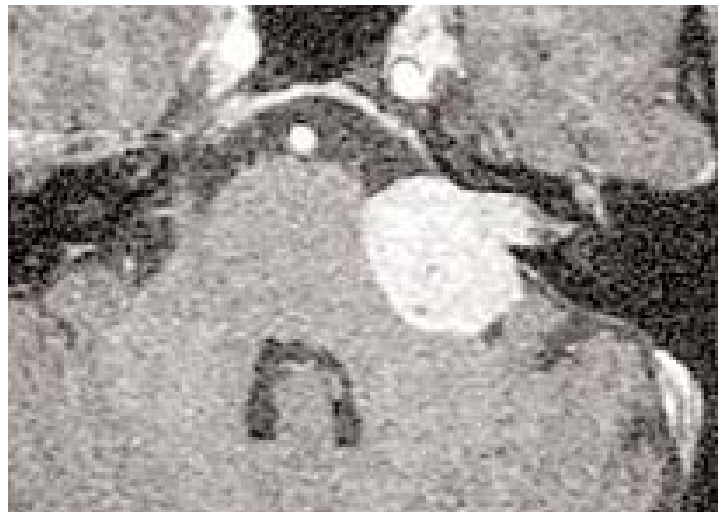

Fig. 3. Axial MR image obtained in a man with a left acoustic neuroma (Survey Case 2).

options. Large tumors with significant brainstem compression usually require resection. For patients with smallor medium-sized tumors, SRS has become a common treatment, with excellent reported long-term results. $\mathrm{Pa}$ tients must be comfortable with the concept of tumor control rather than tumor removal. Most seem to be satisfied with this concept, if it allows them to avoid brain surgery. Surgeons should strive to educate their patients with information from the peer-reviewed literature. Confusion among patients exists because the information provided by internet sources, newsletters, support groups, and physicians has not always been validated and supported by outcomes data. Although we are asked to provide our opinions, our comments should not be based on myth, conjecture, training bias, or socioeconomic concerns.

\section{References}

1. Andrews DW, Suarez O, Goldman HW, et al: Stereotactic radiosurgery and fractionated stereotactic radiotherapy for the treatment of acoustic schwannomas: comparative observations of 125 patients treated at one institution. Int J Radiat Oncol Biol Phys 50:1265-1278, 2001

2. Bederson JB, von Ammon K, Wichmann W, et al: Conservative treatment of patients with acoustic tumors. Neurosurgery 28: 646-651, 1991

3. Brennan JW, Rowed DW, Nedzelski JM, et al: Cerebrospinal fluid leak after acoustic neuroma surgery: influence of tumor size and surgical approach on incidence and response to treatment. J Neurosurg 94:217-233, 2001

4. Cerullo LJ, Grutsch JF, Heiferman K, et al: The preservation of hearing and facial nerve function in a consecutive series of unilateral vestibular nerve schwannoma surgical patients (acoustic neuroma). Surg Neurol 39:485-493, 1993

5. Comey C, McLaughlin MR, Jho HD, et al: Death from a malignant cerebellopontine angle triton tumor despite stereotactic radiosurgery. J Neurosurg 89:653-658, 1998

6. Flickinger JC, Kondziolka D, Lunsford LD: Dose and diameter relationships for facial, trigeminal, and acoustic neuropathies following acoustic neuroma radiosurgery. Radiother Oncol 41:215-219, 1996

7. Flickinger JC, Kondziolka D, Niranjan A, et al: Results of acoustic neuroma radiosurgery: an analysis of 5 years' experience using current methods. J Neurosurg 94:1-6, 2001

8. Flickinger JC, Kondziolka D, Pollock BE, et al: Evolution in technique for vestibular schwannoma radiosurgery and effect on outcome. Int J Radiat Oncol Biol Phys 36:275-280, 1996 
9. Flickinger JC, Lunsford LD, Linskey ME, et al: Gamma knife radiosurgery for acoustic tumors: multivariate analysis of four year results. Radiother Oncol 27:91-98, 1993

10. Flickinger JC, Lunsford LD, Wu A, et al: Treatment planning for gamma knife radiosurgery with multiple isocenters. Int J Radiat Oncol Biol Phys 18:1495-1501, 1990

11. Foote RL, Coffey RJ, Swanson JW, et al: Stereotactic radiosurgery using the gamma knife for acoustic neuromas. Int J Radiat Oncol Biol Phys 32:1153-1160, 1995

12. Gardner G, Robertson JH: Hearing preservation in unilateral acoustic neuroma surgery. Ann Otol Rhinol Laryngol 97: 55-66, 1988

13. Gormley WB, Sekhar LN, Wright DC, et al: Acoustic neuromas: results of current surgical management. Neurosurgery 41:50-60, 1997

14. Hanabusa K, Morikawa A, Murata T, et al: Acoustic neuroma with malignant transformation. Case report. J Neurosurg 95: 518-521, 2001

15. House JW, Brackmann DE: Facial nerve grading system. Otolaryngol Head Neck Surg 93:146-147, 1985

16. Kondziolka D, Dempsey PK, Lunsford LD, et al: A comparison between magnetic resonance imaging and computed tomography for stereotactic coordinate determination. Neurosurgery 30:402-407, 1992

17. Kondziolka D, Lunsford LD, McLaughlin M, et al: Long-term outcomes after radiosurgery for acoustic neuromas. New Engl J Med 339:1426-1433, 1998

18. Linskey ME, Lunsford LD, Flickinger JC: Neuroimaging of acoustic nerve sheath tumors after stereotaxic radiosurgery. AJNR 12:1165-1175, 1991

19. Linskey ME, Lunsford LD, Flickinger JC: Tumor control after stereotactic radiosurgery in neurofibromatosis patients with bilateral acoustic tumors. Neurosurgery 31:829-839, 1992

20. Linskey ME, Martinez AJ, Kondziolka D, et al: The radiobiology of human acoustic schwannoma xenografts after stereotactic radiosurgery evaluated in the subrenal capsule of athymic mice. J Neurosurg 78:645-653, 1993

21. Martin HC, Sethi J, Lang D, et al: Patient-assessed outcomes after excision of acoustic neuroma: postoperative symptoms and quality of life. J Neurosurg 94:211-216, 2001

22. Mazzoni A, Calabrese V, Moschini L: Residual and recurrent acoustic neuroma in hearing preservation procedures: neuroradiologic and surgical findings. Skull Base Surg 6:105-112, 1996

23. Norén G, Arndt J, Hindmarsh T: Stereotactic radiosurgery in cases of acoustic neurinoma: further experiences. Neurosurgery 13:12-22, 1983

24. Ogunrinde OK, Lunsford LD, Flickinger JC, et al: Cranial nerve preservation after stereotactic radiosurgery of small acoustic tumors. Arch Neurol 52:73-79, 1995

25. Ogunrinde OK, Lunsford LD, Flickinger JC, et al: Stereotactic radiosurgery for acoustic tumors in patients with useful preoperative hearing: results at 2-year follow-up examination. J Neurosurg 80:1011-1017, 1994

26. Petit JH, Hudes RS, Chen TT, et al: Reduced-dose radiosurgery for vestibular schwannomas. Neurosurgery 49:1299-1307, 2001
27. Pollock BE, Lunsford LD, Flickinger JC, et al: Vestibular schwannoma management. Part I. Failed microsurgery and the role of delayed stereotactic radiosurgery. J Neurosurg 89: 944-948, 1998

28. Pollock BE, Lunsford LD, Kondziolka D, et al: Outcome analysis of acoustic neuroma management: a comparison of microsurgery and stereotactic radiosurgery. Neurosurgery 36: 215-229, 1995

29. Pollock BE, Lunsford LD, Kondziolka D, et al: Vestibular schwannoma management. Part II. Failed radiosurgery and the role of delayed microsurgery. J Neurosurg 89:949-955, 1998

30. Post KD, Eisenberg MB, Catalano PJ: Hearing preservation in vestibular schwannoma surgery: what factors influence outcome? J Neurosurg 83:191-196, 1995

31. Rowed DW, Nedzelski JM: Hearing preservation in the removal of intracanalicular acoustic neuromas via the retrosigmoid approach. J Neurosurg 86:456-461, 1997

32. Samii M, Matthies C: Management of 1000 vestibular schwannomas (acoustic neuromas): surgical management and results with an emphasis on complications and how to avoid them. Neurosurgery 40:11-23, 1997

33. Samii M, Tatagiba M, Matthies C: Acoustic neurinoma in the elderly: factors predictive of postoperative outcome. Neurosurgery 31:615-620, 1992

34. Shamisa A, Bance M, Nag S, et al: Glioblastoma multiforme occurring in a patient treated with gamma knife surgery. Case report and review of the literature. J Neurosurg 94:816-821, 2001

35. Subach B, Kondziolka D, Lunsford LD, et al: Stereotactic radiosurgery in the management of acoustic neuromas associated with neurofibromatosis Type 2. J Neurosurg 90:815-822, 1999

36. Wiegand DA, Fickel V: Acoustic neuroma-the patient's perspective: subjective assessment of symptoms, diagnosis, therapy, and outcome in 541 patients. Laryngoscope 99:179-187, 1989

37. Wiegand DA, Ojemann RG, Fickel V: Surgical treatment of acoustic neuroma (vestibular schwannoma) in the United States: report from the Acoustic Neuroma Registry. Laryngoscope 106:58-66, 1996

38. Williams JA: Fractionated stereotactic radiotherapy for acoustic neuromas. Stereotact Funct Neurosurg 78:17-28, 2002

39. Yamamoto M, Hagiwara S, Ide M, et al: Conservative management of acoustic neurinomas: prospective study of long-term changes in tumor volume and auditory function. Minim Invasive Neurosurg 41:86-92, 1998

Manuscript received March 3, 2003.

Accepted in final form April 14, 2003.

Address reprint requests to: Douglas Kondziolka, M.D., Department of Neurological Surgery, Suite B-400, University of Pittsburgh Medical Center, 200 Lothrop Street, Pittsburgh, Pennsylvania 15213. email:kondziolkads@msx.upmc.edu. 